\title{
The Kramers Problem: Beyond Quasi-Stationarity
}

\author{
S.M. Soskin ${ }^{1}$, V.I. Sheka ${ }^{1}$, T.L. Linnik ${ }^{1}$, M. Arrayás ${ }^{2}$, \\ I.Kh. Kaufman'², D.G. Luchinsky², P.V.E. McClintock², \\ R. Mannella ${ }^{3}$ \\ ${ }^{1}$ Institute of Semiconductor Physics, Ukrainian Academy of Sciences, Kiev, Ukraine \\ ${ }^{2}$ Department of Physics, Lancaster University, Lancaster, LA1 $4 Y B, U K$ \\ ${ }^{3}$ Dipartimento di Fisica, Università di Pisa, Piazza Torricelli 2, 56100 Pisa, Italy
}

\begin{abstract}
Noise-induced escape from a metastable potential is considered on timescales preceding the formation of quasi-equilibrium within the metastable part of the potential. It is shown that the escape flux may then depend exponentially strongly, and in a complicated manner, on time and friction.
\end{abstract}

\section{INTRODUCTION}

In his seminal work [1], Kramers considered the noise-induced flux from a single metastable potential well i.e. he considered a stochastic system

$$
\begin{aligned}
& \ddot{q}+\Gamma \dot{q}+d U / d q=f(t), \\
& \langle f(t)\rangle=0, \quad\left\langle f(t) f\left(t^{\prime}\right)\right\rangle=2 \Gamma T \delta\left(t-t^{\prime}\right),
\end{aligned}
$$

which was put initially into the bottom of a metastable potential well $U(q)$ and then he calculated the quasi-stationary probability flux beyond the barrier. There have been many developments and generalizations of the Kramers problem (see $[2,3]$ for reviews) but both he and most of those who followed him considered just the quasi-stationary flux, i.e. the flux established after the formation of a quasistationary distribution within the well (up to the barrier). The quasi-stationary flux is characterized by a slow exponential decay, an Arrhenius dependence on temperature $T$, and a relatively weak dependence on friction $\Gamma$ :

$$
J_{q s}(t)=\alpha_{\text {escape }} \mathrm{e}^{-\alpha_{\text {escape }} t}, \quad \alpha_{\text {escape }}=P \mathrm{e}^{-\frac{\Delta U}{T}},
$$

where $P$ depends on $\Gamma$ and $T$ in a non-activation manner.

But how does the flux evolve from its zero value at the initial moment to its quasi-stationary value at time-scales exceeding the time $t_{f}$ for the formation of

CP502, Stochastic and Chaotic Dynamics in the Lakes: STOCHAOS,

edited by D. S. Broomhead, E. A. Luchinskaya, P.V. E. McClintock, and T. Mullin

(C) 2000 American Institute of Physics 1-56396-915-7/00/\$17.00 
quasi-equilibrium? It is obvious that the answer may depend on initial conditions. The most natural are those corresponding to the stable stationary state of the noise-free system i.e. $\left(q=q_{\text {bottom }}, \dot{q}=0\right)$ where $q_{\text {bottom }}$ is the coordinate of the bottom of the potential well. Just such an initial state is assumed in this paper ${ }^{1}$. If the noise is switched on suddenly (e.g. if the thermal isolation of a frozen system is broken) then the time evolution of the escape flux from the noise-free metastable initial state is highly relevant. It might seem natural that the evolution from zero to the quasi-stationary value should be smooth. Such an assumption might also seem to have been confirmed recently by Schneidman [4] who found that, for both the strongly underdamped and overdamped cases, the escape flux from a single metastable well grows with time $t$ smoothly, at $t \sim t_{f}$. But does this exhaust the problem? We prove theoretically, and demonstrate experimentally, that there are two generic situations when the escape flux ${ }^{2}$ behaves in a different manner.

The theoretical approach which we use for this is the method of optimal fluctuation (c.f. e.g. [5]) within which an escape rate is sought in the form

$$
\alpha_{\text {escape }}=P \mathrm{e}^{-\frac{S}{T}}
$$

where the action $S$ does not depend on $T$; the prefactor $P$ does depend on $T$, but relatively weakly. The action $S$ is related to a certain optimal fluctuation which, in turn, corresponds to the most probable escape path (MPEP).

Theoretical results are verified by computer and electronic simulations.

\section{SHORT TIME-SCALES}

The quasi-stationary flux is formed by optimal fluctuations which bring the system from the bottom of the (initial) well to a relevant saddle for an optimal time

$$
t_{\mathrm{opt}} \sim \frac{1}{\min \left(\Gamma, \omega_{0}\right)} \ln \left(\frac{\Delta U}{T}\right)
$$

where $\omega_{0}$ is the frequency of eigenoscillation in the bottom of the well.

At much shorter time-scales,

$$
t \ll t_{\text {opt }}
$$

1) Note that, for the relatively large time-scales considered below in the section for the multiwell case (c.f. Fig. 2), such a restriction is not essential: the results remain valid for any initial distribution within one well. The case of arbitrary initial conditions, for the smaller time-scales as considered in the next section (c.f. Fig. 1), will be explored elsewhere.

2) Our major results are not sensitive to the concrete definition of the flux. It could e.g. be the full flux through a boundary or just a first passage flux; the boundary may e.g. be a given coordinate (as in $[1,3]$ ), or a boundary of a basin of attraction (relevant to an escape rate), or the boundary of a close vicinity to another attractor (relevant to inter-well transition rates). 

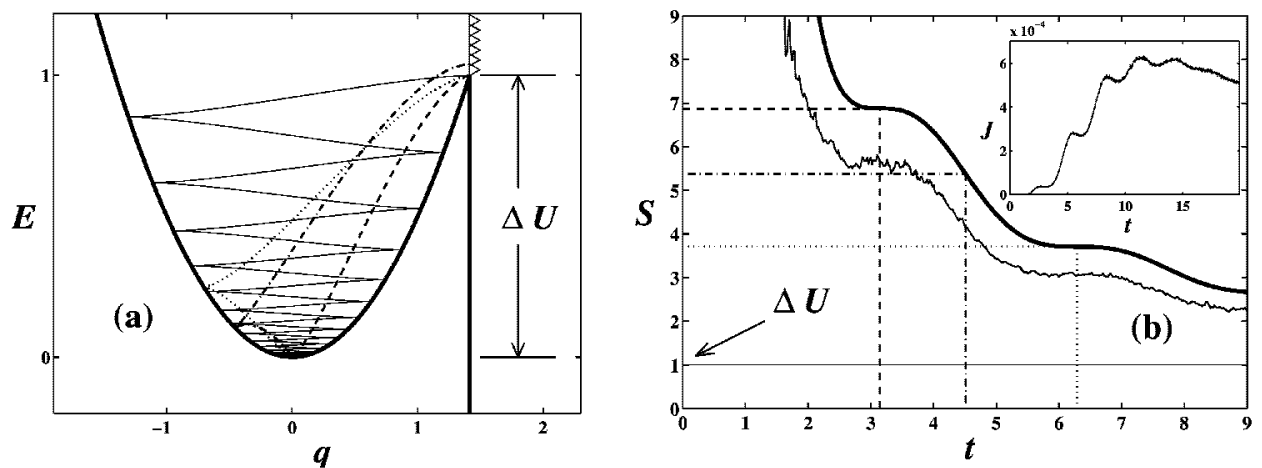

FIGURE 1. (a) Examples of MPEPs (plotted in the energy-coordinate plane $E-q$ where $E=\dot{q}^{2} / 2+U(q)$ ) for an escape from the bottom of the metastable well $U(q)=q^{2} / 2$ at $q<\sqrt{2}$ (thick solid line) to beyond the barrier at $q=\sqrt{2}(U(q)=-\infty$ at $q>\sqrt{2}$, which is equivalent to the absorbing wall indicated by triangles), at $\Gamma=0.05$; (b) the corresponding theoretical (thick solid line) and experimental (thin jagged line) dependences of the action $S$ on the escape time $t$. The dashed and dotted lines indicate: in (b) the 1st and 2nd inflection point with $d S / d t=0$; and in (a) the corresponding MPEPs. The thin solid line shows: in (b) the large-time asymptote level equal to the barrier height $\Delta U$; and in (a) the corresponding MPEP (which is the time-reversal of the noise-free trajectory from the top of the barrier into the bottom of the well). The dash-dotted line shows in (a) the MPEP corresponding to some arbitrarily chosen time $t=4.51$ (see (b)) and demonstrates, in particular, that the escape velocity is generally non-zero. The inset shows the experimental dependence of the flux on time, for $T=1.0$.

the flux is necessarily formed by optimal fluctuations strongly differing from those of duration $t_{\mathrm{opt}}$, and the smaller $t$ the more marked this difference becomes. Thus, in the range (5), $S$ is dependant on $t$. Moreover, we can show rigorously that if $\Gamma<\Gamma_{c}$ where $\Gamma_{c}$ is typically equal to $2 \omega_{0}$ then $S(t)$ is a $s t e p$-wise function: see the example ${ }^{3}$ in Fig. 1. The vertical and horizontal positions of the center of the step $S(t)$ number $n$ (counted from the left) equal respectively $\Delta U \omega_{0} /(n \pi \Gamma)$ and $n \pi / \omega_{0}$, provided $n \pi \Gamma \ll \omega_{0}$. Generally, when the shape of the potential well departs from parabolicity, the equalities turn into approximations. Thus, in the range (5), J depends exponentially strongly both on $\Gamma$ and on $t$ (c.f. the inset).

3) Although we present just one example (chosen for its simplicity), we emphasize that a step-wise dependence of $S(t)$ is typical for any potential, including those which possess a saddle or several wells, and that it remains valid for any reasonable boundary (c.f. footnote 2). We have developed a rigorous (and simple) procedure for obtaining a numerical solution provided the metastable part of the potential is a single well and the absorbing boundary is not too close to a saddle. A qualitative analysis shows that if the boundary is close enough to a saddle (or just includes it) then $S(t)$ possesses folds at certain critical values of $t$ corresponding to switching between MPEPs of different topologies. 

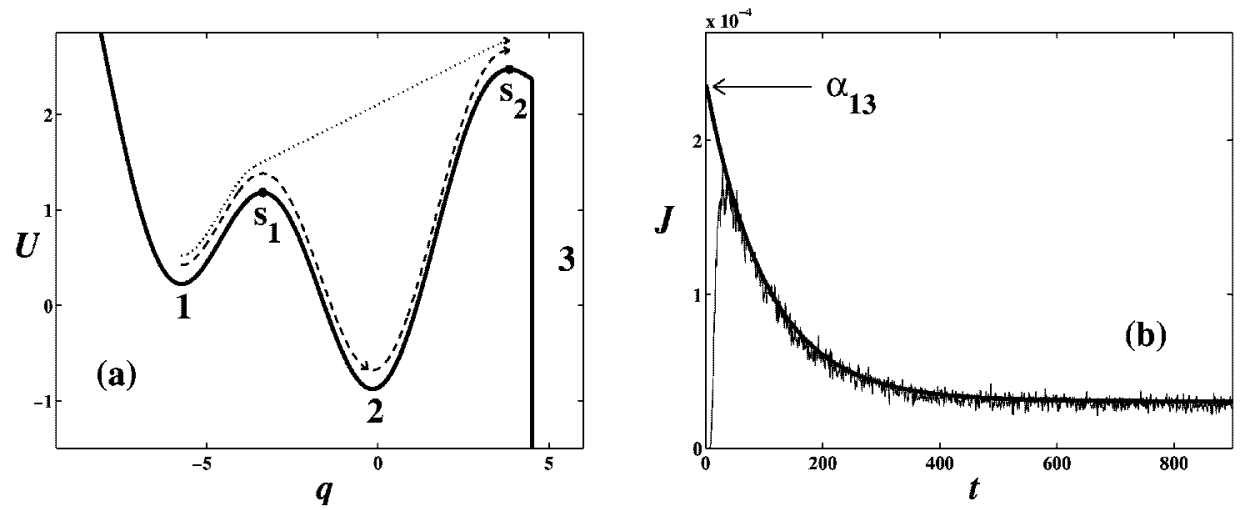

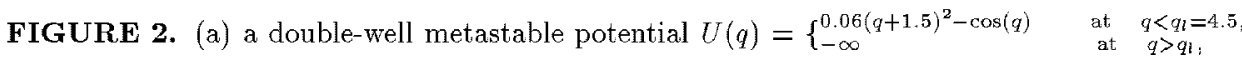
and schematically shown direct (dotted line) and indirect (dashed line) transitions $1 \rightarrow 3$ i.e. escapes from the well 1 beyond the coordinate $q_{l}$; (b) simulations of the escape flux $J(t) 1 \rightarrow q_{l}$ (thin jagged line) at $\Gamma=0.15, T=0.4$, compared with the approximation of $J(t)$ by Eq. (6) (thick full line); $\alpha_{12}, \alpha_{21}$ and $\alpha_{q s}$ are calculated by the Kramers-Melnikov formula [3] while $\alpha_{13}, \alpha_{23}$ are calculated by Eq. (7) with $m \approx 1.1$.

\section{MULTI-WELL METASTABLE POTENTIAL}

Unlike the single-well case, where the formation time of quasi-equilibrium is of the order of $t_{\mathrm{opt}}$ (4), its formation in the multi-well case (e.g. as in Fig. 2) proceeds via two distinct stages: first, quasi-equilibrium is formed within the initial well which as in the single-well case, takes $\sim t_{\mathrm{opt}}$; secondly, quasi-equilibrium between wells becomes established which takes exponentially longer $\left(\sim t_{\mathrm{opt}} \exp (\Delta U / T) \gg t_{\mathrm{opt}}\right.$ where $\Delta U$ means a minimal internal barrier). During the latter stage (as well as during the subsequent quasi-stationary stage), the flux $J(t)$ can be described via a solution of kinetic equations for the well populations, using the concept of constant inter-attractor ${ }^{4}$ transition rates $\alpha_{i j}$ (c.f. [6]):

$$
\begin{aligned}
& J(t)=\alpha_{13} e^{-\frac{t}{t_{s}}}+\alpha_{q s}\left(e^{-\frac{t}{t_{q s}}}-e^{-\frac{t}{t_{s}}}\right), \\
& t_{s} \approx \alpha_{12}^{-1}, \quad t_{q s} \approx \alpha_{q s}^{-1} \approx \alpha_{12} /\left(\alpha_{12} \alpha_{23}+\alpha_{21} \alpha_{13}\right), \\
& T \ll U_{\mathrm{S}_{1}}-U_{1}, \quad t \gg t_{\mathrm{opt}} .
\end{aligned}
$$

The physical meaning of the two terms in (6) is easily understood (c.f. Fig. 2(b)). The first one corresponds to direct escapes (i.e. those ones which do not go via the bottom of well-2) and dominates before the quasi-equilibrium is established; whereas the second term, corresponding to indirect escapes, dominates in the ensuing quasi-stationary stage: it is the asymptotic part of this latter flux, $\alpha_{q s} \exp \left(-t / t_{q s}\right)$, that is called the quasi-stationary flux.

Although the coefficients $\alpha_{12}, \alpha_{21}, \alpha_{q s}$ can readily be obtained from the KramersMelnikov theory [3], $\alpha_{13}$ and $\alpha_{23}$ cannot be found [3] in this way. One of us [7] has developed a theory of $\alpha_{13}, \alpha_{23}$ based on the concept of optimal fluctuation:

4) For the sake of brevity, we refer to region 3 as an "attractor" too. 


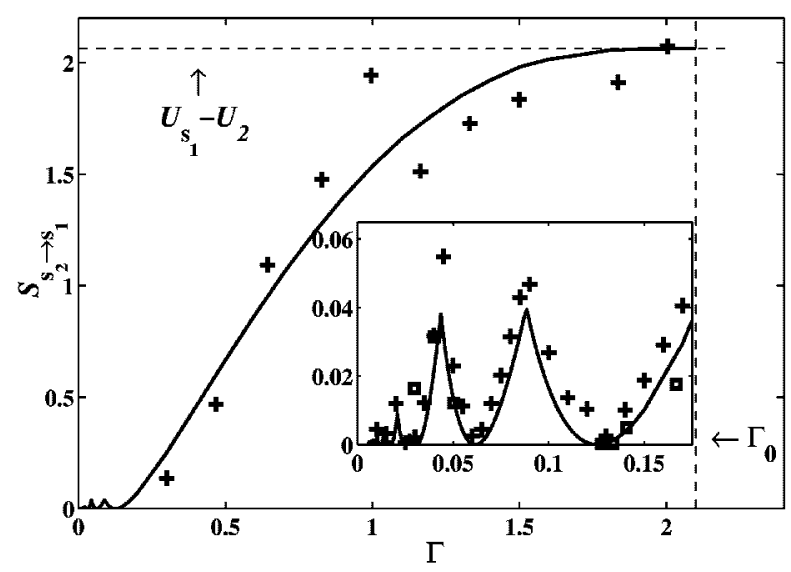

FIGURE 3. Dependence of the action for the transition $s_{2} \rightarrow s_{1}$ on the damping constant $\Gamma$, at time-scales $t \gg t_{\mathrm{opt}}$, in the system (1) with $U(q)$ shown in Fig. 2(a). The solid line is calculated numerically from the theory [7]. The horizontal and vertical dashed lines indicate respectively the upper limit for $S_{\mathrm{s}_{2} \rightarrow \mathrm{s}_{1}}$ and the value of $\Gamma$ at which the cutoff of direct transitions $\mathrm{s}_{2} \rightarrow \mathrm{s}_{1}$ and escapes $1 \rightarrow \mathrm{s}_{2}$ occurs. The crosses and squares represent digital and analogue simulation data respectively. The inset shows an expanded plot of the region of small damping.

$$
\alpha_{13,23}=\alpha_{q s}\left(1+\left(\Omega_{1} \Omega_{2}^{-1} \exp \left(\left(U_{1}-U_{2}\right) / T\right)\right)^{ \pm 1}\right) /\left(1+\left(m \exp \left(k S_{\mathrm{s}_{2} \rightarrow \mathrm{s}_{1}} / T\right)\right)^{ \pm 1}\right),
$$

where: $\Omega_{1}, \Omega_{2}$ are the frequencies of eigenoscillation in the bottom of wells 1,2 ; $k=1$ or -1 for ranges of $\Gamma$ providing the noise-free relaxation from $s_{2}$ respectively into 2 or 1 ; the action $S_{\mathrm{s}_{2} \rightarrow \mathrm{s}_{1}}$ for the transition $\mathrm{s}_{2} \rightarrow \mathrm{s}_{1}$ is calculated from the theory [7]; and $m$ is the only adjustable parameter, related to the prefactor.

As seen from Eq. (7) and Fig. 3, the rates $\alpha_{13}$ and $\alpha_{23}$ depend on friction exponentially strongly, at sufficiently small temperatures. Moreover, they oscillate ${ }^{5}$ in the underdamped range, and $\alpha_{13}$ has a cutoff at a certain friction $\Gamma_{0} \geq 2 \Omega_{2}$ i.e. $\alpha_{13}=0$ for $\Gamma \geq \Gamma_{0}$. The oscillations are related to an alternation between ranges of friction in which a noise-free trajectory from the external saddle $\mathrm{s}_{2}$ goes into either well-1 or well-2: in accordance with the principle of detailed balance, the noise-induced escape $1 \rightarrow s_{2}$ is the time-reversal of $s_{2} \rightarrow 1$, so that its probability is characterized by the Arrhenius factor if a noise-free trajectory $\mathrm{s}_{2} \stackrel{n f}{\rightarrow} 1$ exists; otherwise the probability is exponentially smaller. The cutoff at large $\Gamma$ is related to the absence of turning points in the noise-free trajectories $\mathrm{s}_{1} \stackrel{n f}{\rightarrow} 2, \mathrm{~s}_{2} \stackrel{n f}{\rightarrow} 2$.

\section{CONCLUSIONS AND OPEN PROBLEMS}

We conclude that: (i) escape from a metastable potential differs markedly after and before the formation of quasi-equilibrium within the metastable part of the

5) Typically, the scale of oscillation of $S$ is small in comparison with $U_{\mathrm{s}_{2}}-U_{1}$ (corresponding to the Arrhenius dependence on $T$ ) but, in some cases, it can equal or even exceed $U_{\mathrm{s}_{2}}-U_{1}[7]$. 
potential; (ii) at time-scales much less than the optimal duration of a fluctuation $t_{\text {opt }}$, the escape flux $J$ grows exponentially strongly with time and, moreover, if the friction $\Gamma$ is small, it does so in a step-wise manner and depends exponentially strongly on $\Gamma$; and (iii) if the metastable part of the potential consists of more than one well, then the formation of quasi-equilibrium takes an exponentially long period of time and the escape flux during the most of this period is formed from direct escapes and depends exponentially strongly on friction and, moreover, $J(\Gamma)$ undergoes oscillations in the underdamped range and may drop drastically if $\Gamma$ exceeds a critical value $\Gamma_{0} \geq 2 \Omega_{2}$.

Open problems yet to be addressed include: (i) for the range $t \ll t_{\text {opt }}$ : (a) the transition from a smooth $S(t)$ (with inflection points only) to an $S(t)$ possessing folds, (b) additional features characteristic of the multi-well case, (c) the pre-exponential factor; (ii) the pre-exponential factor for inter-attractor transition rates in the multi-well case; and (iii) a generalization for non-potential systems for which, unlike potential systems where a switching between different MPEPs gives rise only to folds in $S(t)$, we anticipate the possibility of jumps in $S(t)$.

\section{ACKNOWLEDGEMENTS}

The research has been supported by INTAS, and by the Engineering and Physical Sciences Research Council (UK). We are grateful to George Pickett for his assistance with some of the figures.

\section{REFERENCES}

1. Kramers, H.A., Physica 7, 284-304 (1940).

2. Hanggi, P., Talkner, P. and Borkovec, M., Rev. Mod. Phys. 62, 251-341 (1990).

3. Mel'nikov, V.I., Phys. Rep. 209, 1-71 (1991).

4. Shneidman, V.A., Phys. Rev. E 56, 5257-5264 (1997).

5. M. I. Dykman, P. V. E. McClintock, V. N. Smelyanski, N. D. Stein and N. G. Stocks Phys. Rev. Lett. 68, 4547 (1992).

6. Freidlin, M.I. and Wentzell, A.D., Random Perturbations in Dynamical Systems (Springer Verlag, New York, 1984).

7. Soskin, S.M., to appear in J. Stat. Phys. 97, No. 3/4 (1999); see also Los Alamos e-print archive cond-mat/9908432. 
Copyright $\odot 2003$ EBSCO Publishing 
Copyright of AIP Conference Proceedings is the property of American Institute of Physics and its content may not be copied or emailed to multiple sites or posted to a listserv without the copyright holder's express written permission. However, users may print, download, or email articles for individual use. 